\title{
Prandtl-Tietjens intermittency in transitional pipe flows
}

\author{
Rory T. Cerbus:* \\ Université de Bordeaux $\&$ CNRS, LOMA (UMR 5798), 33405 Talence, France
}

(Dated: January 17, 2022)

\begin{abstract}
Pipe flow often traverses a regime where laminar and turbulent flow co-exist. Prandtl and Tietjens explained this intermittency as a feedback between the fluctuations of the internal flow resistance and the constant pressure drop driving the flow. However, because the focus has moved towards studying intermittency without flow fluctuations near the universal critical Reynolds number, their explanation has largely disappeared. Here we refine the mechanism, which has never been put to a quantitative test, to develop a model that agrees with experiments at higher Reynolds numbers, enabling us to demonstrate that Prandtl and Tietjens' mechanism is, in fact, intrinsic to flows where both the pressure gradient and perturbation are constant.
\end{abstract}

In 1839, while investigating the friction in pipe flow, Gotthilf Hagen [1] observed that the jet of water exiting the pipe resembled a glassy rod at low flow speeds, which then began to pulse as the flow speed increased. The jet reflects the state of the flow inside the pipe. It is in one place glassy [1] and smooth [2], "laminar", while frosty [2] and sinuous [3], "turbulent", elsewhere. Hagen's pulses were a manifestation of this intermingling of laminar and turbulent flow, which we now call intermittency, a basic feature of the transition to turbulence in pipe flow and other shear flows [4 8]. The turbulent patches, which can also die, split, or grow, are carried downstream so that the whole pattern of intermittency changes continuously in space and in time. The phenomenon of intermittency was unexpected, given that the flow conditions were kept as constant as practical, and its origin was at first unclear [1 3 , , 9]. In their famous fluid mechanics textbook, Prandtl and Tietjens [10], hereafter referred to as PT, qualitatively explained intermittency as the result of a feedback between the larger friction in the turbulent patches and the constant total pressure drop driving the flow [11]. With a larger friction, the flow speed decreases until it is reduced below the critical speed, so that no new turbulence is created. When the increased friction of the patch leaves the pipe, the flow speed increases. The critical speed is again exceeded, a new patch is created, and the cycle repeats. In the PT mechanism, intermittency not only creates but requires fluctuations in flow speed, both of which oscillate. In keeping with common practice, we will hereafter use the non-dimensional flow speed or Reynolds number, $R e=U D / \nu$, where $U$ is the flow speed, $D$ is the diameter, and $\nu$ is the kinematic viscosity.

The qualitative PT mechanism remained the prevalent explanation until the seminal study of transitional pipe flow by J. Rotta 12]. Rotta accepted the general validity of the PT mechanism but sought to determine if it was the only source of intermittency by taking great pains to maintain an approximately constant $R e$ in his constant pressure drop and constant perturbation experiments [12]. He introduced a large external resistance into his pipe system so that the pressure drop over this resistance would damp out oscillations. Restricting attention to $R e \lesssim 3000$, he found that the intermittency persisted, despite no obvious fluctuations in $R e$, thus demonstrating that the PT narrative does not explain the origin of intermittency everywhere. More recent experiments also use a large resistance [13], and experiments with a constant mass flux [14] have demonstrated convincingly that intermittency can also exist apart from PT's mechanism, although the typical method of instantaneously perturbing the flow renders the experimental initial conditions themselves intermittent. Rotta's insight laid the foundation for studying the patchy, localized turbulence, now believed to originate from special exact solutions of the governing Navier-Stokes equations such as nonlinear traveling waves [4, 5]. Most recent work has focused as Rotta did on the vicinity of the critical Re where non-expanding patches called "puffs" dominate, or considered only instantaneous perturbations at higher Re 13]. Thus, with a few exceptions 11, 15, 16], the PT mechanism has largely disappeared from any discussion of the transition [4, 5, 17]. However, this leaves neglected an important regime of transitional flow, a flow that transitions at $R e \gtrsim 3000$, and for which the pressure gradient and perturbation are constant.

In this Letter we revisit PT's argument and look at the intermittency of transitional pipe flow under essentially constant conditions. It is driven by a constant pressure drop, disturbed continuously, and when not in the transition regime, the variation in $R e, \delta R e$, is small $(\delta R e / R e<0.01)$. We demonstrate the validity of the $\mathrm{PT}$ mechanism by developing a simple model based on their arguments that quantitatively reproduces the essential features of the intermittency in our experiments. Key to the success of our model is accounting for the external resistance, which we systematically vary, as well as accurately incorporating the growth of turbulent patches. The experiments and model together suggest a startling conclusion: under constant conditions and for $R e \gtrsim 3000$, there is always a regime of intermittency consistent with the PT argument.

* rory.cerbus@u-bordeaux.fr 
(a)

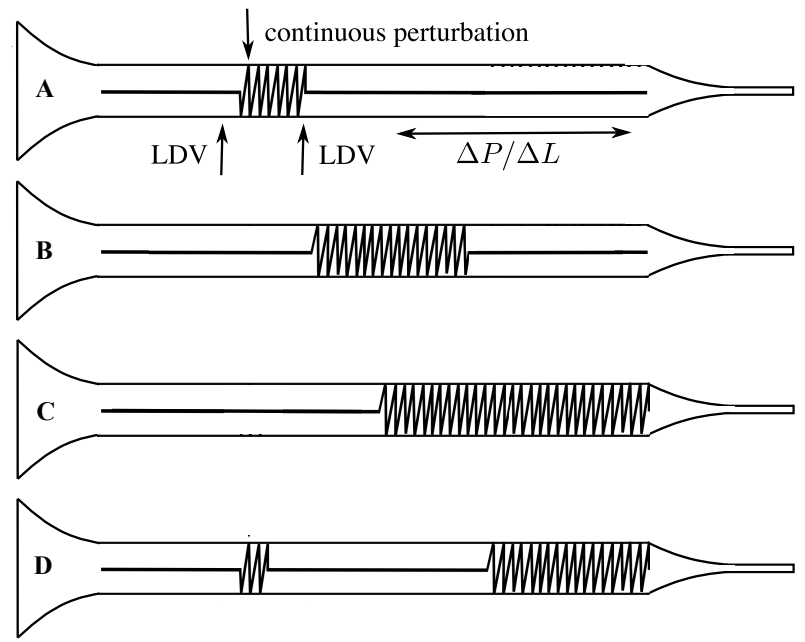

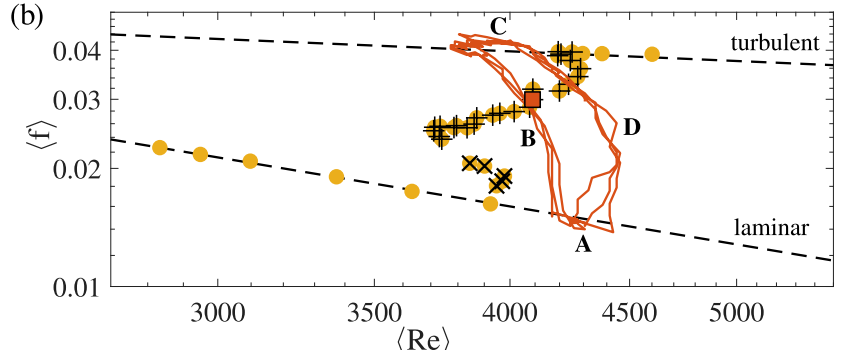

(c)

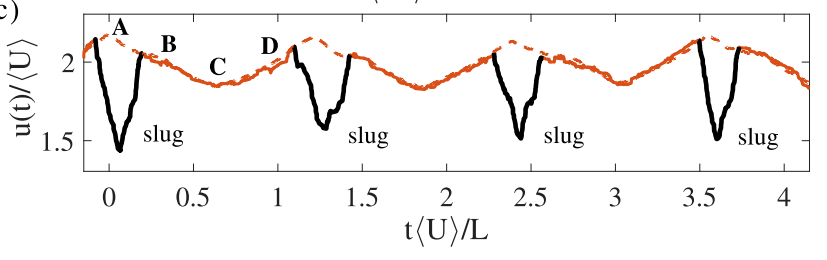

FIG. 1. (a) Schematic of the pipe experiment modelled after that appearing in Reynolds [3], with a contracting entrance section and a final narrow pipe section for added resistance. Flow is from left to right. Straight horizontal lines indicate laminar flow, and jagged flow indicates a slug. The pressure measurement section for determining $\Delta P / \Delta L$ and thus $f$ in (b) is shown, as well as the two positions for measuring the velocity with LDVs in (c). The flow is disturbed continuously with an obstacle to set a transition $R e_{C}$. The flow state at points $\mathbf{A} \rightarrow \mathbf{D}$ from (b) and (c) are represented schematically. (b) Plot of average friction $\langle f\rangle$ vs. average $\langle R e\rangle$. The lower and upper curves are the laminar and turbulent friction curves, respectively. The transitional data are either periodic $(+)$ or stochastic $(\times)$. Overlaid on the mean data $(\circ)$ we plot the instantaneous $f(t)-R e(t)$ curve for one periodic transitional data point $(\square)$. This curve cycles clockwise through the points $\mathbf{A}, \mathbf{B}, \mathbf{C}$, and $\mathbf{D}$. (c) The normalized streamwise velocity $u(t) /\langle U\rangle$ at the centerline vs. the normalized time $t\langle U\rangle / L$ for $\square$, with $\mathbf{A} \rightarrow \mathbf{D}$ also denoted. The velocity was measured $100 \mathrm{D}$ downstream $(-)$ and $10 \mathrm{D}$ upstream (--) from the perturbation. The shape of both curves is the same as $\operatorname{Re}(t)$ but for the slugs (solid black line superimposed over - ).

For our experiments we carry out measurements of the flow rate, velocity, and the friction in a $2020 \mathrm{~cm}$ long, smooth, cylindrical glass pipe of diameter $D=1 \mathrm{~cm} \pm 10 \mu \mathrm{m}$. The working fluid is water. Driven by gravity, the flow remains laminar up to $R e \approx 10000$. We restrict our attention to $3000 \lesssim R e \lesssim 7000$, for which the turbulent patches, called "slugs" 4], grow, an essential ingredient in the PT mechanism. We perturb the flow $\simeq 404 D$ downstream (see Fig. 17) either continuously with an obstacle (a small $\simeq 0.63 \mathrm{~mm}$ diameter rod oriented perpendicular to the flow) or instantaneously with a syringe pump which injects a small amount of fluid from a $1 \mathrm{~mm}$ hole in the pipe wall. We denote by $L$ the distance from the perturbation to the end of the pipe. We can set a natural transition $R e$ when the flow becomes unstable, $R e_{C}$, by adjusting the rod protrusion. (This $R e_{C}$ should not be confused with the lower and universal critical $R e_{C}$ investigated by experiments of puff lifetimes [8].) We determine the instantaneous flow rate using a magnetic flowmeter (Yokogawa) and the total pressure drop $\Delta P_{\text {tot }}$ by measuring the difference between the height of water surface in the source reservoir from the height of the water at the exit of the pipe, $\Delta h$. We also measure the instantaneous pressure drop in a 505D section that is $101 \mathrm{D}$ from the end of the pipe (see Fig. 1 a). Two Laser Doppler velocimeters (LDV, MSE) were also used to probe the flow (see Fig. 19). More experimental details can be found in the Supplementary Material ( $S M$, Sec. II) and in Ref. [18].

We begin by revisiting PT's mechanism through an examination of our experimental data for Re and the nondimensional friction factor $f=D \Delta P / \Delta L /\left(\rho U^{2} / 2\right)$, where $\rho$ is the density, and $\Delta P$ is the pressure drop over a length $\Delta L$. We refer to Fig. 1 b, a traditional plot of $\langle f\rangle$ vs. $\langle R e\rangle$, to investigate the state of the system, where \langle\rangle refers to the time-averaged value. As $\Delta P_{\text {tot }}$ slowly increases (via $\Delta h$ ), the data (o) initially conform to the lower laminar curve, but the flow becomes unstable due to the finite disturbance for $R e>R e_{C} \approx 4000$ (set by the obstacle) and the position of $\langle f\rangle-\langle R e\rangle$ deviates from the laminar curve thereafter. The first slugs appear stochastically $(\times)$ [19], but this behavior spans only a narrow range of $\Delta P_{\text {tot }}$. Thereafter the flow displays periodic behavior $(+)$, which was the original focus of PT and thus ours as well.

In Fig. 10 we plot the instantaneous $f(t)-R e(t)$ curve corresponding to one periodic data point ( $\square)$. To understand this curve, consider the point $\mathbf{A}$ where the flow is laminar. Because $R e(t)>R e_{C} \simeq 4000$, a slug is created by the perturbation and begins to invade the flow, as indicated by a thick black line in Fig. 15 (see also Fig. 19), and it expands aggressively as it is convected downstream [4]. The increased friction with $\Delta P_{\text {tot }}=$ const. requires $R e(t)$ to decrease. The slug eventually reaches the pressure measurement section and partially fills it, raising the value of $f(t)$ to point $\mathbf{B}$, until the flow there is fully turbulent at point $\mathbf{C}$ on the upper curve. As the turbulent patch leaves the pipe, $R e(t)$ increases and the flow's intermittency decreases, taking us through point D (Fig. 19), until finally the flow is fully laminar again and we return to point $\mathbf{A}$ to begin the cycle again. We now attempt to gain further insight 
(a)

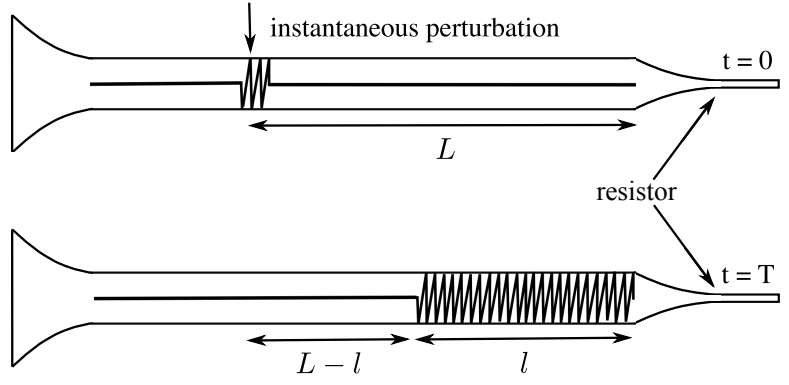

(b) 30

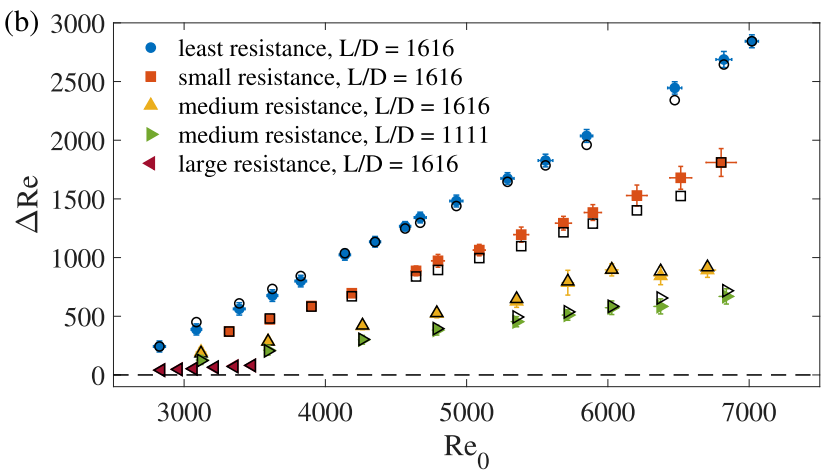

FIG. 2. (a) Schematic of transitional pipe flow as in Fig. 1 A single slug is engendered at $t=0$ by a perturbation, which grows to its maximum size when it reaches the end of the pipe at $t=T$. (b) Plot of deviation in $R e, \Delta R e$, vs. the initial $R e_{0}$ for several resistances $R$. Increasing $R$ or decreasing $L$ reduces $\Delta R e$. A dependence on $L$ is to be expected and is also seen in work on pulsatile flow 20]. The predicted $\Delta R e$, open symbols, are in excellent accord with the experimental data, filled symbols.

by constructing a model to reproduce quantitative features.

We identify four essential ingredients, which we update and refine as necessary. The flow is driven by a constant pressure drop, $\Delta P_{\text {tot }}(\mathcal{I} 1)$, the pressure drop in a turbulent region is higher than a laminar one of the same length $(\mathcal{I} 2)$, slugs are convected and grow $(\mathcal{I} 3)$, and finally, a critical $R e_{C}$ is set by disturbing the flow continuously $(\mathcal{I} 4)$. We first combine $\mathcal{I} 1$ and $\mathcal{I} 2$ by distributing the constant $\Delta P_{\text {tot }}$ between the laminar $\Delta P_{\text {lam }}$ and slug $\Delta P_{\text {turb }}$ portions of the flow. In addition, we also include the pressure drop of the system external to the experimental section, $\Delta P_{\text {ext }}$, contributed by, for example, the entrance section. This gives the pressure drop balance $\Delta P_{\text {tot }}=\Delta P_{\text {lam }}+\Delta P_{\text {turb }}+\Delta P_{\text {ext }}$. As Fig. 10 already indicates, when the pressure measurement region is laminar, $f(t)$ obeys the Hagen-Poiseuille law: $f_{\text {lam }}=64 / R e$, whereas when this region is turbulent, even during transition [18], it obeys the empirical Blasius law: $f_{\text {turb }}=0.3164 R e^{-1 / 4}$. (This allows us to probe intermittency in a straightforward manner: the flow is intermittent if $\left.f_{\text {lam }}<f(t)<f_{\text {turb }}\right)$. We determine $\Delta P_{\text {ext }}$ empirically in a series of experiments when the pipe is fully laminar, $\Delta P_{\text {ext }}=\Delta P_{\text {tot }}-\Delta P_{\text {lam }}$ (see $S M$, Sec. I). Introducing the parameter $l$, the length of the pipe that is turbulent, results in (see $S M$, Sec. I):

$$
\frac{D^{3} \Delta P_{\mathrm{tot}}}{32 \rho \nu^{2} L}=\left(1-\frac{l}{L}\right) R e+B \frac{l}{L} R e^{7 / 4}+R(R e)
$$

where $B=0.3164 / 64$ is a constant combining the constants from the Hagen-Poiseuille and Blasius laws and $R=$ $\frac{D^{3} \Delta P_{\text {ext }}}{32 \rho \nu^{2} L}$ is the normalized external resistance. The terms on the right hand side are the pressure drop contributions from the laminar $(\propto 1-l / L)$, turbulent $(\propto l / L)$ and external portions of the pipe, respectively. Previous work that split $\Delta P_{\text {tot }}$ between a laminar and turbulent contribution also predicted oscillations, but they were unable to show quantitative agreement between model and experiment [15, 16]. This highlights the importance of accounting for the external resistance $R$ and accurately incorporating slug growth rates, both of which were not included in these approaches.

As a first step in validating our refined model, we use Eq. 1 to predict the maximum change in $R e$ when a single slug is created, utilising $\mathcal{I} 1-\mathcal{I} 3$. We perform experiments in which we systematically vary $R$ by adding short sections of smaller diameter pipes (see Fig. 2a), a "resistor", to the pipe system 21, 22] and determine the Re dependence of $R$ empirically (see $S M$, Sec. I). We then perturb the flow instantaneously at a distance $L$ from the end of the pipe where the laminar flow is fully developed. We adjust $\Delta P_{\text {tot }}$ via $\Delta h$ to set an initial $R e=R e_{0}$ and seek the maximum deviation from $R e_{0}: \Delta R e=R e_{0}-R e_{\min }$, where $R e_{\min }$ is the minimum $R e$. For each $\Delta h$ and $R(R e)$ we perform the experiment at least three times to determine averages and uncertainties. For constant $\Delta P_{\text {tot }}$, we can write Eq. 1 at both $R e_{0}$ and $R e_{\min }$ and equate them to show that:

$$
R e_{0}+R\left(R e_{0}\right)=\frac{D^{3} \Delta P_{\mathrm{tot}}}{32 \rho \nu^{2} L}=\left(1-\frac{l}{L}\right) R e_{\min }+B \frac{l}{L} R e_{\min }^{7 / 4}+R\left(R e_{\min }\right),
$$

where for $R e=R e_{0}, l=0$ by definition. The $l / L$, which we next estimate, also depends on $R e$. We suppose that the minimum value $R e_{\min }$ occurs when $l / L$ is at its maximum as the growing slug reaches the end of the pipe. The maximum $l / L$ can be estimated using the slug front speed, $u_{F}$, and back speed, $u_{B}$. If $T$ is the time it takes the slug front to reach the end of the pipe, then $L=u_{F} T$ and $L-l=u_{B} T$, which can be rearranged to find $l / L=\left(u_{F}-u_{B}\right) / u_{F}$. We made our own estimates of $u_{F}$ and $u_{B}$ (see $S M$, Sec. II) because the literature values are 

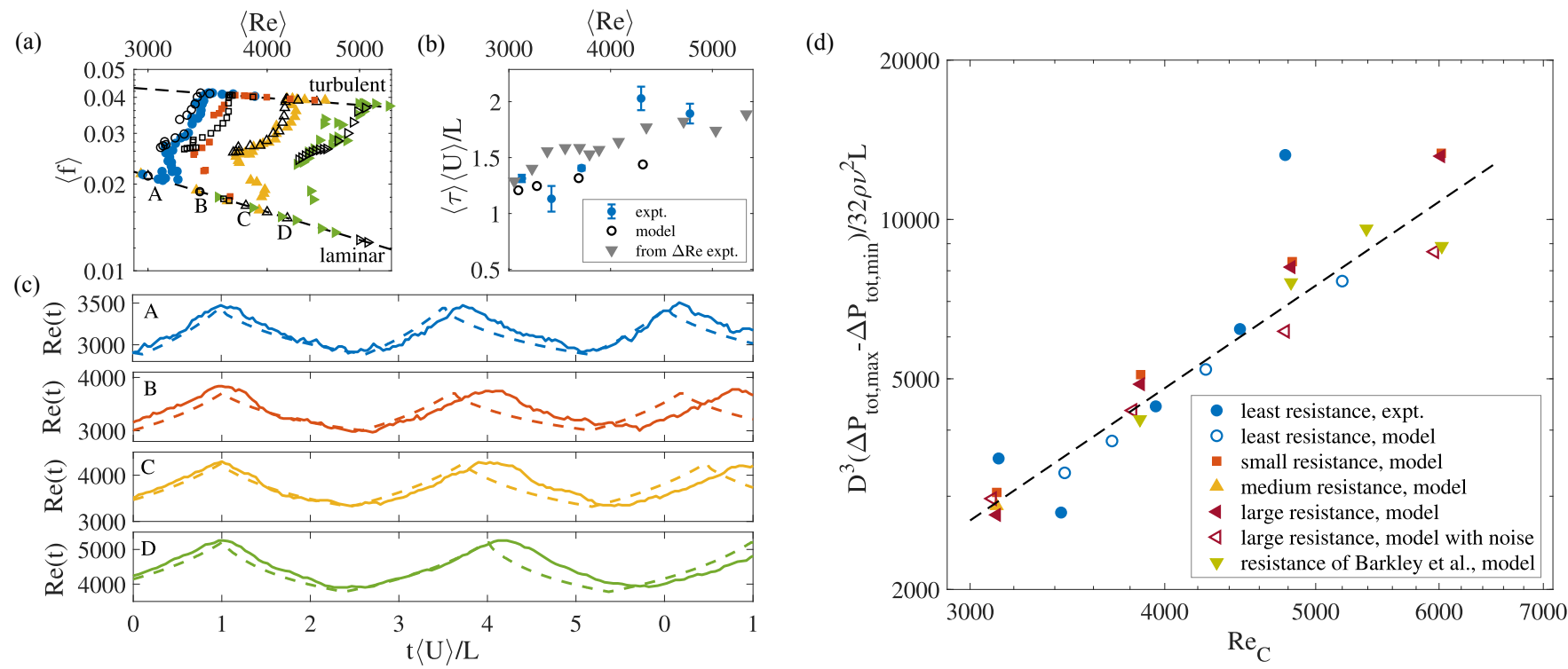

FIG. 3. (a) experiments, filled symbols, and model predictions, open symbols, for $\langle f\rangle$ vs. $\langle R e\rangle$. The model data closely follows the experimental data. For each series of data, $A-D$, we plot in (c) below the normalized time series of $R e(t)$ vs. $t\langle U\rangle / L$, choosing the point of minimum $\langle R e\rangle$ in the periodic intermittent regime. The amplitudes and normalized periods $\tau\langle U\rangle / L$ closely match the experiments. (b) $\tau\langle U\rangle / L$ determined from experiments, the model, and from the previous $\Delta R e$ experiments (Fig. 2 $\tau\langle U\rangle / L \simeq\langle U\rangle / u_{B}$ ). The normalized periods differ by $\lesssim 5 \%$ in most cases. (d) Non-dimensional intermittency span vs. transition $R e_{C}$ for experiments and model simulations ( $R$ as in Fig. 2). We also include model simulations using an extremely large $R$ estimated from Barkley et al.'s experiments [13] (see $S M$, Sec. V for details). The ratio of smallest to largest resistance is $\simeq 500$ and the $L / D$ varied up to $\simeq 24 \%$. We also probed the effect of external noise by adding normally distributed noise with zero mean and standard deviation of 0.1 to $R e(t)$ at each step in the integration of Eq. 3 Despite these differences, all data collapse onto a common curve and exhibit a non-negligible span of intermittency that increases with $R e_{C}\left(\propto R e_{C}^{2},--\right)$.

for practically constant $R e$ [13, 23 25]. Because the external resistance in these experiments is deliberately smaller, the Re here is not constant. We then solve Eq. 2 numerically, and Fig. 20 shows that its predictions are in excellent accord with the experimental results. The variation in the $R e$ as the slug grows also leads to a subtle dependence on the pipe length $L$, as the growing slug has more time to slow down the flow if $L$ is larger. Thus as Fig. $2 \mathrm{~b}$ shows, for the same external resistance but smaller $L / D, \Delta R e$ is smaller.

We now proceed to develop a time-dependent version of the model to reproduce the oscillations, now incorporating a critical $\operatorname{Re}_{C}(\mathcal{I} 4)$. We take the time derivative of Eq. $1(\mathcal{I} 1, \mathcal{I} 2)$, subject to the constraint $\Delta P_{\text {tot }}=$ const. $(\mathcal{I} 1)$, which yields:

$$
\frac{d R e}{d t}=\frac{\frac{d(l / L)}{d t}\left(R e-B R e^{7 / 4}\right)}{\left(1-\frac{l}{L}\right)+\frac{7}{4} B R e^{3 / 4}\left(\frac{l}{L}\right)+\frac{d R}{d R e}} .
$$

To determine the time-dependence of $l / L$ we use a recent model which has had significant success in reproducing the growth rates $(\mathcal{I} 3)$ of slugs [13]. The complexity of slug growth is reduced to two coupled partial differential equations for a variable representing the turbulence intensity, $q$, and the pipe centerline velocity $u$. Now together with Eq. 3 we have a set of coupled partial and ordinary differential equations. Since the $l / L$ in Eq. 3 is simply the total turbulent fraction, we do not use the spatial information of the partial differential equations in Eq. 3 This system of equations is similar to, but simpler than, the systems of coupled differential equations used to model arterial flow [26].

We perform several experiments without an external resistor, although $R \neq 0$, systematically changing the transition $R e_{C}$ by adjusting the amplitude of the perturbation (I2). For each $R e_{C}$, set by adjusting the obstacle, we repeated the experiment of Fig. 1 $\mathrm{b}$, slowly increasing $\Delta P_{\text {tot }}$ to take the system from laminar, to intermittent, to turbulent (see Fig. 33). For our model, we integrate Eq. 3 along with the coupled partial differential equations from Barkley et al.'s model [13], which we transformed into laboratory units (see $S M$, Sec. III). To reproduce the behavior in our experiments we add a constant perturbation to the Barkley model, the amplitude of which we varied to set different transition $R_{C}$ as in the experiments $(\mathcal{I} 4)$. This deterministic model is not able to reproduce the initial region of intermittency, in which slugs appear stochastically, but it both quantitatively reproduces the oscillations and the shapes of the $\langle f\rangle$ vs. $\langle R e\rangle$ curves (see Fig. 3r).

As Figs. 2, 3a, 3b, 3r show, our model, based on the PT mechanism, is in excellent accord with the experimental data. We now use this result to demonstrate the generality of the PT mechanism. As already noted, Rotta tested 
the PT mechanism by restricting attention near the universal critical point $(R e \lesssim 3000)$ and by increasing the external resistance. The former invalidates the PT mechanism because it removes slug growth, an essential ingredient (I3). As Fig. 2b demonstrates, the latter approach of increasing $R$ unsurprisingly reduces deviations in $R e(\Delta R e)$. Indeed, this principle is broadly used to maintain a nearly constant $R e$ in constant pressure gradient transitional pipe flow experiments. If fluctuations can be completely eliminated, one would expect no intermittency and thus in our $\langle f\rangle-\langle R e\rangle$ curves there would be a discontinuous jump from the laminar to turbulent friction curves at $R e_{C} \gtrsim 3000$. To test the hypothesis that the regime of intermittency shrinks as $R$ increases, we plot versus $R e_{C}$ in Fig. $3 \mathrm{~d}$ the normalized difference between the pressure drop at the end of the intermittent regime $\Delta P_{\text {tot,max }}$ and at the beginning $\Delta P_{\text {tot,min }}$. Despite spanning over two orders of magnitude in $R$, and even in the presence of noise, all data collapse onto a single curve that inexplicably increases with $R e_{C}$. When expressed in terms of the true control variable, the normalized pressure gradient, the intermittency span is independent of $R$. Moreover the attendant intermittency is not negligible, since the fraction of flow filled by patches necessarily advances continuously from zero to unity

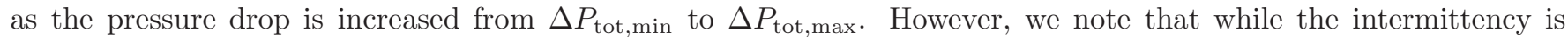
substantial, the relative magnitude of the fluctuations in $R e$ can be substantially reduced by increasing $R$, as shown in Fig. 2b. Near the natural transition point $R e_{C}$ the finite-amplitude threshold is very sharp and thus very sensitive for $R e \gtrsim 3000$ [7, 27], so that even these small variations in $R e$ are sufficient for the PT mechanism to function. Prandtl-Tietjens intermittency is thus an intrinsic feature of continuously perturbed and constant pressure driven flows, for which substantial intermittency and tunable fluctuations in $R e$ are unavoidable if $R e>R e_{C}>3000$.

In conclusion, we have developed a model inspired by Prandtl and Tietjens' classic argument that is in excellent quantitative agreement with experiments. Essential to the model's success was accurately accounting for the external resistance and slug growth rates. We began our inquiry by noting that, beginning with Rotta [12], the Prandtl-Tietjens argument has been considered irrelevant. Together, our experiments and model suggest that intermittency engendered by the Prandtl-Tietjen mechanism is in fact an intrinsic feature of constant pressure driven pipe flow for constant conditions, and for $R e \gtrsim 3000$. Rotta did not avoid it by increasing the resistance in his pipe, which ultimately cannot remove the intermittency engendered by the PT mechanism (Fig. [3]), but by restricting attention to $R e \lesssim 3000$ [12], just as many other laboratory experiments restrict attention to $R e<R e_{C}$ [8, 13, 25] in order to consider the effect of instantaneous perturbations. Thus while the PT mechanism elucidated here does not apply to those important studies, neither do they directly address the intermittency in the early experiments of Hagen [1], Brillouin [28], and others [6], or those conducted here. Most pipes will have a natural transition $R e_{C}$ set by imperfections such as wall roughness [29, 30], and here it is the Prandtl-Tietjens mechanism which provides the route to turbulence. Fusing old insights [10] and new [13, 18] has broadened the impact of both, yielding new and practical understanding of transitional pipe flow.

Acknowledgments. We thank Tom Mullin for suggesting this problem, as well as Pinaki Chakraborty and Hamid Kellay for helpful discussions. We thank an anonymous referee for correcting our interpretation of Rotta's paper in an earlier draft. We thank the Service Informatique at the Laboratoire Ondes et Matière d'Aquitaine for computational support. R.T.C. gratefully acknowledges the support of a Marie Skłodowska-Curie Action Individual Fellowship (MSCAIF), and the support of the Okinawa Institute of Science and Technology (OIST) where the experiments were carried out.

[1] G. Hagen, Ueber die bewegung des wassers in engen cylindrischen röhren, Annalen der Physik 122, 423 (1839).

[2] M. Couette, Distinction de deux régimes dans le mouvement des fluides, Journal de Physique Théorique et Appliquée 9, 414 (1890).

[3] O. Reynolds, An experimental investigation of the circumstances which determine whether the motion of water shall be direct or sinuous, and of the law of resistance in parallel channels, Proceeds of the Royal Society of London 35, 84-99 (1883).

[4] T. Mullin, Experimental studies of transition to turbulence in a pipe, Annual Review of Fluid Mechanics 43, 1 (2011).

[5] B. Eckhardt, T. M. Schneider, B. Hof, and J. Westerweel, Turbulence transition in pipe flow, Annu. Rev. Fluid Mech. 39, 447 (2007).

[6] C. Letellier, Intermittency as a transition to turbulence in pipes: A long tradition from reynolds to the 21st century, Comptes Rendus Mécanique 345, 642 (2017).

[7] Y. Tasaka, T. M. Schneider, and T. Mullin, Folded edge of turbulence in a pipe, Physical Review Letters 105, 174502 (2010).

[8] K. Avila, D. Moxey, A. de Lozar, M. Avila, D. Barkley, and B. Hof, The onset of turbulence in pipe flow, Science 333, 192 (2011).

[9] L. Sackmann, Sur les changements de régime dans les canalisations - étude cinématographique de la transition, Comptes Rendus Hebdomadaires des Seances de L'Academie des Sciences 239, 220 (1954).

[10] O. K. G. Tietjens and L. Prandtl, Applied hydro-and aeromechanics, Vol. 2 (Courier Corporation, 1957) pages 36-38. 
[11] D. J. Tritton, Physical fluid dynamics (Springer Science \& Business Media, 2012).

[12] J. Rotta, Experimenteller beitrag zur entstehung turbulenter strömung im rohr, Ingenieur-Archiv 24, 258 (1956).

[13] D. Barkley, B. Song, V. Mukund, G. Lemoult, M. Avila, and B. Hof, The rise of fully turbulent flow, Nature 526, 550 (2015).

[14] A. Darbyshire and T. Mullin, Transition to turbulence in constant-mass-flux pipe flow, Journal of Fluid Mechanics 289, 83 (1995).

[15] D. Stassinopoulos, J. Zhang, P. Alstrøm, and M. T. Levinsen, Periodic states in intermittent pipe flows: Experiment and model, Physical Review E 50, 1189 (1994).

[16] A. C. Fowler and P. Howell, Intermittency in the transition to turbulence, SIAM Journal on Applied Mathematics 63, 1184 (2003).

[17] D. Barkley, Theoretical perspective on the route to turbulence in a pipe, J. Fluid Mech 803 (2016).

[18] R. T. Cerbus, C.-C. Liu, G. Gioia, and P. Chakraborty, Laws of resistance in transitional pipe flows, Physical Review Letters 120, 054502 (2018).

[19] J. Zhang, D. Stassinopoulos, P. Alström, and M. T. Levinsen, Stochastic transition intermittency in pipe flows: Experiment and model, Physics of Fluids 6, 1722 (1994).

[20] D. Xu and M. Avila, The effect of pulsation frequency on transition in pulsatile pipe flow, Journal of Fluid Mechanics 857, 937 (2018).

[21] D. Samanta, A. De Lozar, and B. Hof, Experimental investigation of laminar turbulent intermittency in pipe flow, Journal of Fluid Mechanics 681, 193 (2011).

[22] A. De Lozar and B. Hof, An experimental study of the decay of turbulent puffs in pipe flow, Philosophical Transactions of the Royal Society A: Mathematical, Physical and Engineering Sciences 367, 589 (2009).

[23] E. R. Lindgren, Propagation velocity of turbulent slugs and streaks in transition pipe flow, The Physics of Fluids $\mathbf{1 2}, 418$ (1969).

[24] I. J. Wygnanski and F. Champagne, On transition in a pipe. part 1. the origin of puffs and slugs and the flow in a turbulent slug, Journal of Fluid Mechanics 59, 281 (1973).

[25] M. Nishi, B. Ünsal, F. Durst, and G. Biswas, Laminar-to-turbulent transition of pipe flows through puffs and slugs, Journal of Fluid Mechanics 614, 425 (2008).

[26] P. Reymond, F. Merenda, F. Perren, D. Rufenacht, and N. Stergiopulos, Validation of a one-dimensional model of the systemic arterial tree, American Journal of Physiology-Heart and Circulatory Physiology 297, H208 (2009).

[27] B. Hof, A. Juel, and T. Mullin, Scaling of the turbulence transition threshold in a pipe, Physical review letters 91, 244502 (2003).

[28] M. Brillouin, Leçons sur la viscosité des liquides et des gaz, Vol. 1 (Gauthier-Villars, 1907).

[29] D. Cotrell, G. B. McFadden, and B. Alder, Instability in pipe flow, Proceedings of the National Academy of Sciences 105, 428 (2008).

[30] J. Tao, Critical instability and friction scaling of fluid flows through pipes with rough inner surfaces, Physical Review Letters 103, 264502 (2009). 